\title{
Exploitation of necroptosis for treatment of caspase-compromised cancers (Review)
}

\author{
YOUNG SIK CHO and HEY LI PARK \\ College of Pharmacy, Keimyung University, Daegu 704-701, Republic of Korea
}

Received May 10, 2016; Accepted June 30, 2016

DOI: $10.3892 / 01.2017 .6285$

\begin{abstract}
Programmed necrosis, or necroptosis, is a type of specialized cell death with necrotic characteristics, including the loss of membrane integrity and swollen organelles in dying cells. However, unlike simple necrosis, it may be induced as an alternative form of cell death when apoptosis is blocked and it is mediated in an orchestrated manner, similar to apoptosis, by a series of signaling molecules. Necroptosis-associated proteins and their specific small molecules have been extensively identified in order to illuminate the underlying mechanisms by which necroptosis is activated through a novel signaling pathway. However, the biological significance of necroptosis, which is known as a secondary route of apoptosis, remains under debate. Concurrent with these concerns, the clinical application of necroptosis has been cautiously proposed to treat necroptosis-associated diseases, and to overcome resistance to anticancer drugs. Accordingly, the present review will highlight the harnessing of necroptosis for anticancer therapy. To this end, the state-of-the art technique of necroptosis as a cancer therapy will be briefly described, and then its potential for clinical purposes will be delineated. For a further understanding of necroptosis, the present review begins with a basic introduction to necroptosis and its multifaceted physiological consequences.
\end{abstract}

\section{Contents}

1. Introduction

2. Physiological roles and pathophysiological conditions associated with necroptosis

3. Therapeutic exploitation of necroptosis

4. Perspectives of necroptosis

Correspondence to: Professor Young Sik Cho, College of Pharmacy, Keimyung University, 1000 Sindang-dong, Daegu 704-701, Republic of Korea

E-mail: yscho123@kmu.ac.kr

Key words: Programmed necrosis, necroptosis, anticancer therapy, apoptosis

\section{Introduction}

To maintain cellular homeostasis, cell survival is in a state of constant equilibrium with cell death (1). When cell demise occurs in an ordered and controlled way to cause programmed cell death, this is termed apoptosis. As well as being part of normal tissue turnover, apoptosis is an essential process for development, differentiation and immune responses (2-4). It is known to be actively involved in the removal of useless or severely damaged cells (5). In contrast, necrosis is a type of unrequired cell death that occurs when cells are exposed to overwhelming stresses, including radiation overdose or toxic chemicals. Concurrently, there is an ordered type of necrosis that is executed by signaling pathway of its associated proteins. This type of programmed cell death, which exhibits necrotic features, is termed programmed necrosis or necroptosis. It was initially considered to be a specialized and regulated form of necrotic cell death (6). At present, necroptotic cell death is known to serve a central function in cell development, immunity, cancer and degenerative diseases (7-10). It exhibits typical necrotic characteristics and is under the control of a well-defined signaling pathway. The present review begins with a description of necroptosis-derived features, and updated information on necroptosis regulators and their specific inhibitors.

A list of features discriminating between apoptosis and necroptosis are summarized in Table I.Cells undergoing apoptosis are morphologically shrunken with condensed cytoplasm, while necroptotic cells and nuclei are swollen. Membrane integrity is a definite determinant parameter to discriminate apoptosis and necroptosis (11). Cells that are dying by apoptosis or necroptosis exhibit intact or disintegrated membranes, respectively. At the molecular level, a cascade of signaling pathways leading to caspase activation is required for the mediation of apoptosis via intrinsic or extrinsic pathways, but necroptosis is achieved by the formation of the receptor-interacting protein kinase (RIP)1-RIP3 necrosome complex (12). Necroptosis is different from apoptosis as the former exhibits more marked physiological effects compared with the latter. Specifically, necroptotic cells release intracellular danger signaling molecules into the media to provoke inflammation and immune responses. These endogenous molecules are referred to as damage-associated molecular patterns (DAMPs), which include high mobility group box 1 (HMGB1), DNA fragments and mitochondrial contents. In particular, HMGB1 is a major DAMP protein derived from necroptotic cells, and serves a pivotal role in 
triggering inflammatory responses (11). Apoptotic cells are completely cleared by macrophages or neighboring cells, therefore presenting no apparent physiological responses when compared with the consequences of necroptosis. Conclusively, the physical and biochemical parameters that characterize apoptosis or necroptosis contribute to different physiological outcomes in biological systems.

The terminology 'programmed' indicates that each processing step is developed in a well-organized way and specifically regulated in an orchestrated manner. Necroptosis-associated proteins have been extensively identified through RNA interference screening to establish a series of signaling networks (13). Notably, certain key regulators are known to execute tumor necrosis factor $\alpha(\mathrm{TNF} \alpha)$-mediated necroptosis. The signaling pathway leading to necroptosis is summarized in Fig. 1. Upon TNFa ligation to its cognate TNF $\alpha$ receptor (TNFR), RIP1, TNF receptor 1-associated death domain protein, Fas-associated death domain and caspase-8 are assembled to form complex I (14). Subsequently, transition of the membrane-bound complex I to the cytosolic complex II ensues, leaving TNFR (15). Then, a tumor suppressor cylindromatosis (CYLD) protein promotes the deubiquitination of RIP1 in either complex I or complex II $(16,17)$. It is generally hypothesized that necroptosis is mediated by formation of the RIP1-RIP3 complex when caspase is defective (18). Since the identification of RIP3 as a proximal protein of necroptosis, a mitochondrial protein phosphoglycerate mutase family member 5 (PGAM5) and mixed lineage kinase domain-like (MLKL) protein have been additionally identified as downstream proteins of TNFR ligation. The RIP1-RIP3 complex transmits a death signal to its downstream target, MLKL $(19,20)$. MLKL has been suggested to be responsible for reactive oxygen species generation and c-Jun N-terminal kinase activation during $\mathrm{TNF} \alpha$-induced necroptosis (20). PGAM5, an additional protein that interacts with RIP3, has been identified in addition to RIP1 and MLKL (21). The PGAM5 gene encodes two protein isoforms, PGAM5-long form and PGAM5-short form, via alternative splicing (22). One of these splice variants, PGAM5-short form, may recruit mitochondrial fission factor dynamin-related protein 1 to cause mitochondrial fragmentation (21). PGAM5 functions at the convergence point of multiple necrotic death pathways, linking extracellular stimuli derived from $\mathrm{TNF} \alpha$ to the mitochondria through ligation of the death receptor and activation of a series of intracellular proteins (21). With the identification of necroptosis-associated proteins, a few small molecules that may specifically modulate necroptosis have been identified via high-throughput screening (23). Necrostatin-1 (Nec-1) and necrosulfonamide are specific inhibitors of RIP1 and MLKL, respectively $(23,24)$. These are valuable chemical probes to confirm the presence of necroptotic cell death and to elucidate the underlying molecular mechanisms. Extensive identification of specific necroptotic proteins with the development of specific small molecules may provide data to fill the gaps in these signaling pathways.

\section{Physiological roles and pathophysiological conditions associated with necroptosis}

Originally, necroptosis was regarded as an alternative cell death modality to apoptosis upon $\mathrm{TNF} \alpha$ stimulation. Its activation and subsequent release of DAMPs may not only serve a physiological function, but also cause a wide range of diseases (Table II). Necroptotic cell death is distinctive from necrosis in the sense that the cells actively respond to death stresses, and is also hypothesized to be a method of cell demise when apoptosis is compromised. Genome-wide small interfering RNA analysis demonstrated that a set of 432 genes were enriched in the immune and nervous systems, and that the cellular response to necroptosis was affected by a signaling network relevant to innate immunity (13). At present, necroptosis is proposed to be the dominant cell death program when apoptosis is inhibited (25). Generally, apoptosis via the intrinsic or extrinsic pathways has been regarded as the primary mechanism for the contraction phase of $\mathrm{T}$ cell immunity and the elimination of autoreactive $\mathrm{T}$ cells. However, necroptosis serves a key function in maintaining $\mathrm{T}$ cell homeostasis with defective B-cell lymphoma-2-like protein 11, which is a crucial effector in the negative selection of autoreactive thymocytes, highlighting that caspase inactivation leads to induction of necroptosis (26). In addition, the death of host cells through necroptosis contributes to the first defense mechanism against infectious pathogens that may suppress or evade apoptotic surveillance. In fact, cells infected with viruses may be removed by apoptotic cell death or the immune response. When a virus disarms the apoptotic machinery of host cells for the proliferation of its progeny, necroptosis may be induced as an alternative form of cell death to inhibit virus propagation (27). In addition, RIP3-mediated necroptosis provides a secondary process to clear pathogens through the induction of inflammation (12). Intracellular pathogens, including Mycobacterium tuberculosis and Salmonella typhimurium, transduce type I interferon signaling to kill macrophages via the induction of necroptosis $(28,29)$. As demonstrated by the dynamic functions of necroptosis during viral or bacterial infection, the excess of intracellular molecules from cells undergoing necrosis or necroptosis leads to a pro-inflammatory response, which may provoke the immune system to fight against pathogens.

Conversely, excessive necroptosis in peripheral tissues and ischemia reperfusion injury may cause an inflammatory signal that leads to detrimental consequences, which may result from the release of DAMPs from necroptotic cells into the extracellular compartment. In a previous study, 33 out of 432 genes identified were proposed to be implicated in human diseases, including Huntington's disease, although the associations between necroptosis-regulating genes and human diseases have remained elusive (13). There is a growing body of evidence suggesting that necroptosis is associated with pathological conditions including acute pancreatitis, retinal detachment, renal ischemic reperfusion injury, myocardial infarction and traumatic brain injury (Table II) (12,26-36). Necroptotic cell death was identified in cerulein-induced acute pancreatitis, in which RIP3 overexpression was induced in the pancreas but not in other areas (30). In addition, necroptosis of Paneth cells in the terminal ileum was revealed to be associated with the pathogenesis of inflammatory bowel disease (31). There is also marked RIP3 expression in patients with inflammatory disorders (31). In addition, necroptotic cell death is actively induced in photoreceptor cell loss and acute kidney injury $(32,33)$. A notable previous study suggested that the inhibition of necroptosis was protective against 
Table I. Key features discriminating apoptosis and necroptosis.

Feature or characteristic

Cell \& organelles morphology

Membrane integrity

DNA ladder fragmentation

Signaling pathway

Molecular complex

Biological markers

Physiological significance
Apoptosis

Necroptosis

\author{
Swelling \\ Disintegrated \\ No \\ RIP1/RIP3/MLKL/PGAM5 \\ Necrosome \\ High mobility group B1 \\ Inflammation and innate immunity
}

RIP, receptor-interacting protein kinase; MLKL, mixed lineage kinase domain-like; PGAM5, phosphoglycerate mutase family member 5.

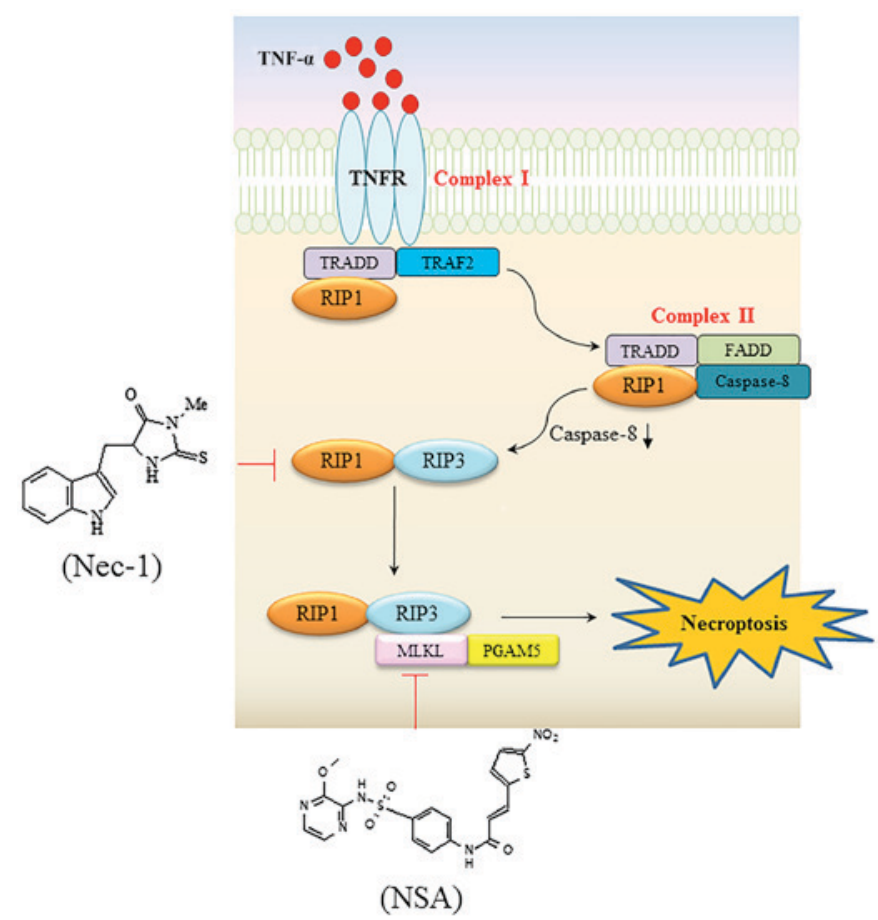

Figure 1. Signaling pathway resulting in TNF $\alpha$-mediated necroptosis, and specific necroptotic inhibitors targeting RIP1 and MLKL. Upon TNF $\alpha$ binding to its cognate receptor, TRADD, TRAF2 and RIP1 are recruited to form complex I. In the second step, bound proteins dissociate from the receptor when TNFR is engulfed into the cytosol. In turn, TRADD and RIP1 are bound to FADD and caspase 8, eventually forming the cytoplasmic complex II. In situations where caspase is compromised, RIP1 interacts with RIP3 to trigger consecutive downstream signaling events, including the recruitment of MLKL and PGAM5, which transmit cytosolic death signals to the mitochondria. Nec-1 and NSA inhibit RIP1 and MLKL, respectively, with high specificity. TNF $\alpha$, tumor necrosis factor $\alpha$; RIP, receptor-interacting protein kinase; MLKL, mixed lineage kinase domain-like; TRADD, TNF receptor 1-associated death domain protein; TRAF2, TNK receptor-associated factor 2; TNFR, TNF $\alpha$ receptor; FADD, Fas-associated death domain; PGAM5, phosphoglycerate mutase family member 5; Nec-1, necrostatin-1; NSA, necrosulfonamide.

liver-associated disease conditions (34). Acetaminophen may induce RIP3 expression along with elevated levels of alanine aminotransferase (ALT) in mice, leading to extensive necrosis. Wild type mice subjected to morpholino antisense targeting RIP3, or RIP3-deficient mice, are protected against acetaminophen-induced liver damage (34). The activation of necroptosis has previously been demonstrated to be a prerequisite for the pathogenesis of multiple sclerosis (35). Kitur et al (36) revealed that the toxin derived from Staphylococcus aureus caused necroptosis-associated lung damage.

\section{Therapeutic exploitation of necroptosis}

As described above, necroptosis is a specialized cell death mode that is does not occur in normal homeostasis. It may be induced by external stresses in conjunction with specific circumstances involving the absence of caspase. Necroptosis has previously been documented in multiple diseases, including ischemic brain injury and degenerative diseases (10). In addition, it becomes an alternative cell death mechanism in multicellular organisms when cells are infected with pathogens that are able to evade the apoptotic machinery of the host. This process is associated with the innate immune response, and may be the first line of defense against pathogens, including viruses and bacteria. Understanding the molecular mechanisms by which necroptosis may be activated is of significance for the implementation of a protective strategy against microbial infection. As well as the defensive function of necroptosis in the host, attention has been paid to harnessing alternative cell death pathways to fight tumor cells with acquired resistance to cancer drugs. Along with apoptosis, necroptosis is a promising cell death process for sensitizing tumor cells to anticancer drugs, and its induction is expected to be a therapeutic tool for killing tumor cells, particularly apoptosis-resistant types of cancer. Cancer cells may evolve to multiply by evading chemotherapy-induced apoptosis, whilst remaining inherently susceptible to necroptosis (37). Therefore, exploitation of the induction of necroptosis may be a secondary therapy to counteract types of cancer resistant to apoptosis. The potential induction of necroptosis for cancer therapy has been paradoxically encouraged by the fact that necroptosis is impaired during tumorigenesis (38). Chronic lymphocytic leukemia cells exhibit defects in necroptosis regulators, including RIP3 and CYLD, an enzyme that may regulate RIP1 ubiquitination (39). RIP3 polymorphisms in non-Hodgkin lymphoma have been demonstrated to be correlated with tumor progression (40). Therapeutically, necroptosis should be induced or suppressed for anticancer therapy or the prevention of necroptosis-associated pathological diseases, respectively. For a more selective induction of necroptosis, 


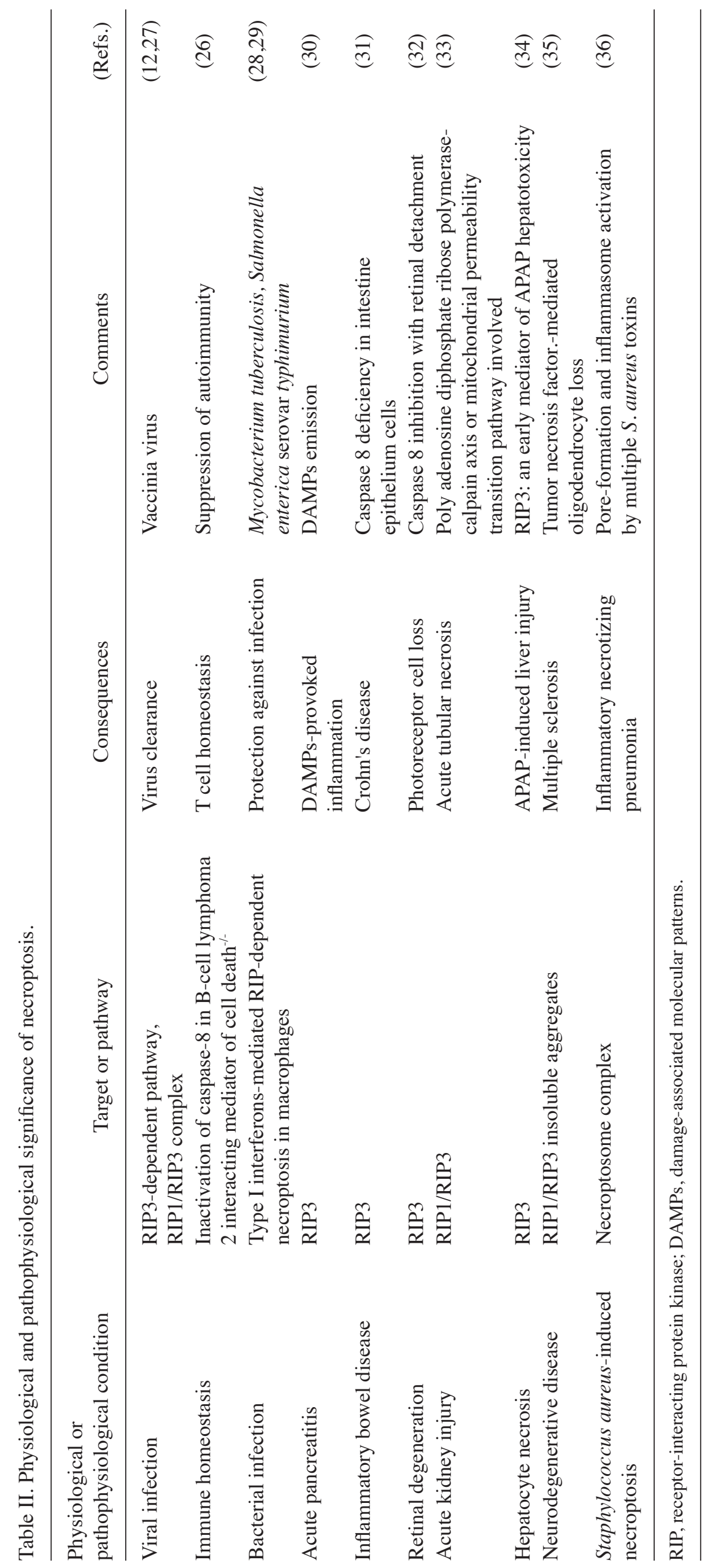


the construction of signaling pathways connecting necroptosis proteins and the development of small molecules that may target necroptosis regulators are required. However, this becomes difficult when considering the interplay between apoptosis, necrosis and autophagy-associated networks. Accordingly, the intricate molecular crosstalk between death modalities should be fully understood prior to the clinical application of necroptosis.

There are a number of feasible examples in which the induction of necroptosis may be applied as a cancer treatment. For example, a number of incurable cancers, including lung cancer, have evolved to evade or interfere with apoptotic machinery when challenged with repetitive treatment with anticancer drugs (41). Accordingly, as an alternative cell death pathway, necroptosis may be exploited to control cancer cells with acquired anticancer drug resistance. The combined treatment of a caspase inhibitor and an antagonist of inhibitor of apoptosis proteins triggers TNF $\alpha$-induced necroptosis in various apoptosis-resistant cell lines and patient xenografts (42). Ovarian cancer cells undergoing necroptosis exhibit the formation of a necrosome-like complex with RIP1 (42). This suggests that it is feasible to target the necroptotic signaling pathway identified in ovarian cancer cells in a therapeutic setting. In addition, Table III summarizes the synthetic small molecules or natural products that may trigger necroptotic cell death in cancer cells. Obatoclax-bearing indole bipyrrole moiety is able to induce necroptosis via the formation of the necrosome on the autophagosomal membrane (43). Staurosporin, a protein kinase inhibitor, and B12536, which targets mitotic kinase pololike kinase 1 , have been suggested to induce necroptosis $(44,45)$. A synthetic naftopidil analogue, HUHS1015, kills human gastric cancer cells via the induction of necroptosis and caspase-independent apoptosis (46). A amiloride derivative, 5'-betaenzylglycinyl-amiloride, was identified to be an inducer of caspase-independent necroptosis in glioma cells (47). The Chinese medicine shikonin promotes necroptotic cell death of glioma cells in a RIP1-dependent manner (48). Shikonin has also been suggested to exert antitumor effects on osteosarcoma by inducing RIP1- and RIP3-dependent necroptosis (49). The use of an additional natural compound, honokiol, in combination with chemotherapeutic agents synergistically kills drug-resistant cell lines via apoptosis and necroptosis (50). As well as chemotherapy, photodynamic therapy using a photosensitizer talaporfin sodium has been indicated to mediate necroptotic cell death in glioblastoma T98 G cells via a signaling pathway consisting of RIP1, RIP3 and MLKL (51). In addition, radiotherapy was performed to induce necroptosis in anaplastic thyroid and adrenocortical cancers (52). Specifically, Nec-1 and zVAD effectively protect cells from radiotherapy, indicating that necroptosis is partly involved in radiation-induced cell death.

\section{Perspectives of necroptosis}

As aforementioned, necroptosis was initially considered a secondary cell death pathway to TNF $\alpha$-induced apoptosis under a caspase-deficient condition. At present, it is hypothesized that necroptosis may be triggered to evoke physiological and pathological consequences to diverse stimuli, although its regulatory mechanism remains unknown. From physiological and pathological aspects, its activation may be beneficial or harmful depending on the stimulus context, and on the cell-specific responses to it. Therefore, the induction of necroptosis may not only provide a secondary safety mechanism against pathogenic infection, but may also be associated with various diseases: Apart from innate immune surveillance, an increasing number of diseases associated with necroptosis, including tissue inflammation and degeneration, have been identified. Necroptosis has been demonstrated to be involved in various neurological disorders, including trauma, strokes, multiple sclerosis and Huntington's disease (53). In addition, the conversion of cholesterol to 24(S)-hydroxycholesterol and its consequent passage through blood-brain barrier is hypothesized to induce necroptosis in neuronal cells that are caspase-8-defective (54). Therefore, protection against necroptotic cell death is of primary concern to prevent the pathogenesis of these diseases. Genetic or pharmacological interference with necroptosis signaling results in neuroprotection against ischemic heart or brain injury $(53,55)$. RIP3 deficiency or administration of Nec-1 has been demonstrated to exhibit protective effects on necroptosis-based heart or brain damage $(55,56)$. Apart from RIP3, additional potent target proteins have been identified as regulators of necroptotic cell death, including RIP1, MLKL, PGAM5 and CYLD (17,21,23,57), which comprise a cascade of signaling pathways for necroptosis (Fig. 1). Subsequently, a small number of inhibitors targeting RIP1 or MLKL have been developed to effectively protect against necroptotic cell death $(23,57)$. Hence, additional identification and validation of more potential targets will be crucial for the development of drugs that may improve pathological conditions.

Beyond necroptosis-associated pathological consequences, necroptosis may be exploited as an alternative therapy to overcome drug-resistant types of cancer. It is based on the hypothesis that a failure in cancer management may be caused by the acquired ability of cancer cells to evade cancer drug-induced apoptosis. Therefore, inducing necroptosis may kill cancer cells and improve immune responses to the danger molecules derived from dying cells, although the release of intracellular contents from dying cells may also promote neoplasia. Although not described in the present review, autophagy complicates the processes of cell death or survival depending on the cell types or the context of the stress. For example, exposure to Obatoclax in rhabdomyosarcoma results in substantial autophagy, which in turn causes RIP3 activation and then necroptotic cell death (43). Notably, caspase-8 does not inhibit RIP3 activation in the autophagosome-driven necroptotic process, unlike receptor-mediated necroptosis (43). In this situation, Obatoclax mediates necroptosis by forming necrosome complexes on autophagosomes. These data highlight that understanding the crosstalk between necroptosis and other cell death types is a prerequisite for selecting optimal treatments customized to specific types of cancer and necroptosis-associated diseases. In conclusion, the comprehensive regulation of cell death is expected to provide clinical opportunities to use cell death programs to treat various diseases, including different types of cancer and degenerative disorders. 


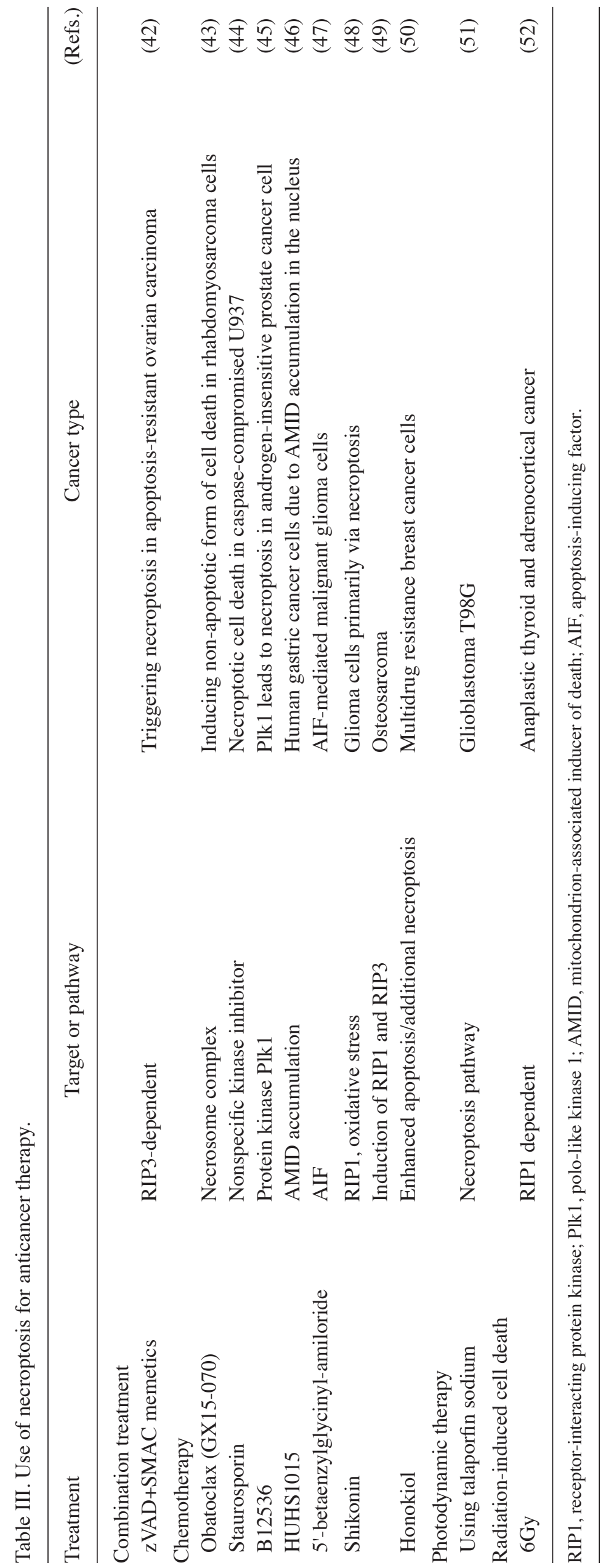




\section{References}

1. Fulda S, Gorman AM, Hori O and Samali A: Cellular stress responses: Cell survival and cell death. Int J Cell Biol 2010: 214074,2010

2. Haanen C and Vermes I: Apoptosis: Programmed cell death in fetal development. Eur J Obstet Gynecol Reprod Biol 64 129-133, 1996

3. Opferman JT: Apoptosis in the development of the immune system. Cell Death Differ 15: 234-242, 2008

4. Duval D, Trouillas M, Thibault C, Dembelé D, Diemunsch F Reinhardt B, Mertz AL, Dierich A and Boeuf H: Apoptosis and differentiation commitment: Novel insights revealed by gene profiling studies in mouse embryonic stem cells. Cell Death Differ 13: 564-575, 2006

5. Elliott MR and Ravichandran KS: Clearance of apoptotic cells: Implications in health and disease. J Cell Biol 189: 1059-1070, 2010.

6. Vanden Berghe T, Linkermann A, Jouan-Lanhouet S, Walczak H and Vandenabeele P: Regulated necrosis: The expanding network of non-apoptotic cell death pathways. Nat Rev Mol Cell Biol 15: $135-147,2014$.

7. Giampietri C, Starace D, Petrungaro S, Filippini A and Ziparo E: Necroptosis: Molecular signalling and translational implications. Int J Cell Biol 2014: 490275, 2014

8. Lu JV, Chen HC and Walsh CM: Necroptotic signaling in adaptive and innate immunity. Semin Cell Dev Biol 35: 33-39, 2014.

9. Fulda S: The mechanism of necroptosis in normal and cancer cells. Cancer Biol Ther 14: 999-1004, 2013.

10. Zhou W and Yuan J: Necroptosis in health and diseases. Semin Cell Dev Biol 35: 14-23, 2014.

11. Kaczmarek A, Vandenabeele P and Krysko DV: Necroptosis: The release of damage-associated molecular patterns and its physiological relevance. Immunity 38: 209-223, 2013.

12. Cho YS, Challa S, Moquin D, Genga R, Ray TD, Guildford M and Chan FK: Phosphorylation-driven assembly of the RIP1-RIP3 complex regulates programmed necrosis and virus-induced inflammation. Cell 137: 1112-1123, 2009.

13. Hitomi J, Christofferson DE, Ng A, Yao J, Degterev A, Xavier RJ and Yuan J: Identification of a molecular signaling network that regulates a cellular necrotic cell death pathway. Cell 135 $1311-1323,2008$.

14. Festjens N, Vanden Berghe T, Cornelis S and Vandenabeele P RIP1, a kinase on the crossroads of a cell's decision to live or die. Cell Death Differ 14: 400-410, 2007.

15. Micheau O and Tschopp J: Induction of TNF receptor I-mediated apoptosis via two sequential signaling complexes. Cell 114 181-190, 2003.

16. Han J, Zhong CQ and Zhang DW: Programmed necrosis: Backup to and competitor with apoptosis in the immune system. Nat Immunol 12: 1143-1149, 2011.

17. Moquin DM, McQuade T and Chan FK: CYLD deubiquitinates RIP1 in the TNF $\alpha$-induced necrosome to facilitate kinase activation and programmed necrosis. PLoS One 8: e76841, 2013.

18. Moriwaki K and Chan FK: RIP3: A molecular switch for necrosis and inflammation. Genes Dev 27: 1640-1649, 2013.

19. Sun L, Wang H, Wang Z, He S, Chen S, Liao D, Wang L, Yan J, Liu W, Lei X and Wang X: Mixed lineage kinase domain-like protein mediates necrosis signaling downstream of RIP3 kinase. Cell 148: 213-227, 2012.

20. Zhao J, Jitkaew S, Cai Z, Choksi S, Li Q, Luo J and Liu ZG Mixed lineage kinase domain-like is a key receptor interacting protein 3 downstream component of TNF-induced necrosis. Proc Natl Acad Sci USA 109: 5322-5327, 2012.

21. Wang Z, Jiang H, Chen S, Du F and Wang X: The mitochondrial phosphatase PGAM5 functions at the convergence point of multiple necrotic death pathways. Cell 148: 228-243, 2012.

22. Remijsen Q, Goossens V, Grootjans S, Van den Haute C, Vanlangenakker N, Dondelinger Y, Roelandt R, Bruggeman I, Goncalves A, Bertrand MJ, et al: Depletion of RIPK3 or MLKL blocks TNF-driven necroptosis and switches towards a delayed RIPK1 kinase-dependent apoptosis. Cell Death Dis 5: e1004, 2014

23. Degterev A, Hitomi J, Germscheid M, Ch'en IL, Korkina O, Teng X, Abbott D, Cuny GD, Yuan C, Wagner G, et al: Identification of RIP1 kinase as a specific cellular target of necrostatins. Nat Chem Biol 4: 313-321, 2008.

24. Liao D, Sun L, Liu W, He S, Wang X and Lei X: Necrosulfonamide inhibits necroptosis by selectively targeting the mixed lineage kinase domain-like protein. Med Chem Comm 5: 333-337, 2014.
25. Christofferson DE and Yuan J: Necroptosis as an alternative form of programmed cell death. Curr Opin Cell Biol 22: 263-268, 2010.

26. Bohgaki T, Mozo J, Salmena L, Matysiak-Zablocki E, Bohgaki M, Sanchez O, Strasser A, Hakem A and Hakem R: Caspase-8 inactivation in $\mathrm{T}$ cells increases necroptosis and suppresses autoimmunity in Bim-/-mice. J Cell Biol 195: 277-291, 2011

27. Upton JW, Kaiser WJ and Mocarski ES: Virus inhibition of RIP3-dependent necrosis. Cell Host Microbe 7: 302-313, 2010.

28. Desvignes L, Wolf AJ and Ernst JD: Dynamic roles of type I and type II IFNs in early infection with Mycobacterium tuberculosis. J Immunol 188: 6205-6215, 2012.

29. Robinson N, McComb S, Mulligan R, Dudani R, Krishnan L and Sad S: Type I interferon induces necroptosis in macrophages during infection with Salmonella enterica serovar Typhimurium. Nat Immunol 13: 954-962, 2012

30. He S, Wang L, Miao L, Wang T, Du F, Zhao L and Wang X: Receptor interacting protein kinase-3 determines cellular necrotic response to TNF-alpha. Cell 137: 1100-1111, 2009.

31. Gunther C, Martini E, Wittkopf N, Amann K, Weigmann B, Neumann H, Waldner MJ, Hedrick SM, Tenzer S, Neurath MF and Becker C: Caspase- 8 regulates TNF- $\alpha$-induced epithelial necroptosis and terminal ileitis. Nature 477: 335-339, 2011.

32. Trichonas G, Murakami Y, Thanos A, Morizane Y, Kayama M, Debouck CM, Hisatomi T, Miller JW and Vavvas DG: Receptor interacting protein kinases mediate retinal detachment-induced photoreceptor necrosis and compensate for inhibition of apoptosis. Proc Natl Acad Sci USA 107: 21695-21700, 2010.

33. Linkermann A, De Zen F, Weinberg J, Kunzendorf U and Krautwald S: Programmed necrosis in acute kidney injury. Nephrol Dial Transplant 27: 3412-3419, 2012.

34. Ramachandran A, McGill MR, Xie Y, Ni HM, Ding WX and Jaeschke H: Receptor interacting protein kinase 3 is a critical early mediator of acetaminophen-induced hepatocyte necrosis in mice. Hepatology 58: 2099-2108, 2013.

35. Ofengeim D, Ito Y, Najafov A, Zhang Y, Shan B, DeWitt JP, Ye J, Zhang X, Chang A, Vakifahmetoglu-Norberg $\mathrm{H}$, et al: Activation of necroptosis in multiple sclerosis. Cell Rep 10: $1836-1849,2015$

36. Kitur K, Parker D, Nieto P, Ahn DS, Cohen TS, Chung S, Wachtel S, Bueno S and Prince A: Toxin-induced necroptosis is a major mechanism of Staphylococcus aureus lung damage. PLoS Pathog 11: e1004820, 2015

37. Su Z, Yang Z, Xie L, DeWitt JP and Chen Y: Cancer therapy in the necroptosis era. Cell Death Differ 23: 748-756, 2016.

38. Meng MB, Wang HH, Cui YL, Wu ZQ, Shi YY, Zaorsky NG, Deng L, Yuan ZY, Lu Y and Wang P: Necroptosis in tumorigenesis, activation of anti-tumor immunity, and cancer therapy. Oncotarget 7: 57391-57413, 2016.

39. Liu P, Xu B, Shen W, Zhu H, Wu W, Fu Y, Chen H, Dong H, Zhu Y, Miao K, et al: Dysregulation of TNFo-induced necroptotic signaling in chronic lymphocytic leukemia: Suppression of CYLD gene by LEF1. Leukemia 26: 1293-1300, 2012.

40. Wu W, Liu P and Li J: Necroptosis: An emerging form of programmed cell death. Crit Rev Oncol Hemato 82: 249-258, 2012.

41. Housman G, Byler S, Heerboth S, Lapinska K, Longacre M, Snyder N and Sarkar S: Drug resistance in cancer: An overview. Cancers (Basel) 6: 1769-1792, 2014.

42. McCabe KE, Bacos K, Lu D, Delaney JR, Axelrod J, Potter MD, Vamos M, Wong V, Cosford ND, Xiang R and Stupack DG: Triggering necroptosis in cisplatin and IAP antagonist-resistant ovarian carcinoma. Cell Death Dis 5: e1496, 2014.

43. Basit F, Cristofanon S and Fulda S: Obatoclax (GX15-070) triggers necroptosis by promoting the assembly of the necrosome on autophagosomal membranes. Cell Death Differ 20: 1161-1173, 2013.

44. Dunai ZA, Imre G, Barna G, Korcsmaros T, Petak I, Bauer PI and Mihalik R: Staurosporine induces necroptotic cell death under caspase-compromised conditions in U937 cells. PLoS One 7: e41945, 2012

45. Deeraksa A, Pan J, Sha Y, Liu XD, Eissa NT, Lin SH, Yu-Lee LY: Plk1 is upregulated in androgen-insensitive prostate cancer cells and its inhibition leads to necroptosis. Oncogene 32: 2973-2983, 2013.

46. Kaku Y, Tsuchiya A, Kanno T and Nishizaki T: HUHS1015 induces necroptosis and caspase-independent apoptosis of MKN28 human gastric cancer cells in association with AMID accumulation in the nucleus. Anticancer Agents Med Chem 15: 242-247, 2015 
47. Pasupuleti N, Leon L, Carraway KL III and Gorin F: 5-Benzylglycinyl-amiloride kills proliferating and nonproliferating malignant glioma cells through caspase-independent necroptosis mediated by apoptosis-inducing factor. J Pharmacol Exp Ther 344: 600-615, 2013.

48. Huang C, Luo Y, Zhao J, Yang F, Zhao H, Fan W and Ge P: Shikonin kills glioma cells through necroptosis mediated by RIP-1. PLoS One 8: e66326, 2013.

49. Fu Z, Deng B, Liao Y, Shan L, Yin F, Wang Z, Zeng H, Zuo D, Hua $\mathrm{Y}$ and Cai Z: The anti-tumor effect of shikonin on osteosarcoma by inducing RIP1 and RIP3 dependent necroptosis. BMC Cancer 13: 580, 2013.

50. Tian W, Deng Y, Li L, He H, Sun J and Xu D: Honokiol synergizes chemotherapy drugs in multidrug resistant breast cancer cells via enhanced apoptosis and additional programmed necrotic death. Int J Oncol 42: 721-732, 2013.

51. Miki Y, Akimoto J, Moritake K, Hironaka C and Fujiwara Y: Photodynamic therapy using talaporfin sodium induces concentration-dependent programmed necroptosis in human glioblastoma T98G cells. Lasers Med Sci 30: 1739-1745, 2015.

52. Nehs MA, Lin CI, Kozono DE, Whang EE, Cho NL, Zhu K Moalem J, Moore FD Jr and Ruan DT: Necroptosis is a novel mechanism of radiation-induced cell death in anaplastic thyroid and adrenocortical cancers. Surgery 150: 1032-1039, 2011.
53. Liu T, Bao YH, Wang Y and Jiang JY: The role of necroptosis in neurosurgical diseases. Braz J Med Biol Res 48: 292-298, 2015.

54. Yamanaka K, Saito Y, Yamamori T, Urano Y and Noguchi N: 24(S)-hydroxycholesterol induces neuronal cell death through necroptosis, a form of programmed necrosis. J Biol Chem 286: 24666-24673, 2011.

55. Luedde M, Lutz M, Carter N, Sosna J, Jacoby C, Vucur M, Gautheron J, Roderburg C, Borg N, Reisinger F, et al: RIP3, a kinase promoting necroptotic cell death, mediates adverse remodelling after myocardial infarction. Cardiovasc Res 103: 206-216, 2014

56. King MD, Whitaker-Lea WA, Campbell JM, Alleyne CH Jr and Dhandapani KM: Necrostatin-1 reduces neurovascular injury after intracerebral hemorrhage. Int J Cell Biol 2014: 495817, 2014.

57. Wang H, Sun L, Su L, Rizo J, Liu L, Wang LF, Wang FS and Wang X: Mixed lineage kinase domain-like protein MLKL causes necrotic membrane disruption upon phosphorylation by RIP3. Mol Cell 54: 133-146, 2014. 